\title{
Repair of orbital wall defects using biocoral scaffolds combined with bone marrow stem cells enhanced by human bone morphogenetic protein -2 in a canine model
}

\author{
CAIWEN XIAO $^{1 *}$, HUIFANG ZHOU $^{1 *}$, SHENGFANG GE $^{1}$, TINGTING TANG $^{2}$, \\ HONGLIANG HOU ${ }^{2}$, MIN LUO $^{1}$ and XIANQUN FAN ${ }^{1}$ \\ Departments of ${ }^{1}$ Ophthalmology and ${ }^{2}$ Orthopedics, Shanghai Ninth People's Hospital, \\ Shanghai JiaoTong University School of Medicine, Shanghai, P.R. China
}

Received April 26, 2010; Accepted June 28, 2010

DOI: 10.3892/ijmm_00000494

\begin{abstract}
Repair of orbital bone defects caused by trauma, infection or cancer is a continuous challenge in reconstructive surgery. Few studies have reported the application of tissue engineering for the repair of orbital bone defects in large animal models. Thus, we investigated the effects of tissueengineered bone enhanced by the human bone morphogenetic protein-2 (BMP2) on the repair of orbital wall defects in a canine model. Autologous bone marrow stromal cells (BMSCs) from 16 Beagle dogs were isolated and cultured in vitro. Passage 2 cells were transfected with adenovirus containing human BMP2 (adv-BMP2) and tissue-engineered bone was constructed using BMP2-expressing BMSCs seeded on a biocoral scaffold. Circular defects (12-mm diameter) created bilaterally in the canine medial orbital wall, were treated with one of the following: adv-BMP2-transfected $\mathrm{BMSC} /$ coral composite (group I, $\mathrm{n}=8$ ), BMSC/coral composite (group II, n=8), biocoral alone (group III, n=8), or were left untreated (group IV, n=8). Four samples from each group were harvested at 12 and 24 weeks after surgery, and the volume and density of newly regenerated bone were determined by micro-computed tomographic (micro-CT) measurement. The rate of new bone deposition and regeneration was measured by tetracycline/calcein labeling and histomorphometric analysis. The results showed that a canine 12-mm circular orbital defect was a critical-sized defect, and the micro-CT and histomorphometry detection results indicated that the combined delivery of BMSCs and BMP2 (group I) resulted
\end{abstract}

Correspondence to: Professor Xianqun Fan, Department of Ophthalmology, Shanghai Ninth People's Hospital, Shanghai JiaoTong University School of Medicine, Shanghai, P.R. China

E-mail: drfanxianqun@126.com

${ }^{*}$ Contributed equally

Key words: tissue engineering, bone morphogenetic protein-2, bone marrow stromal cells, medial orbital wall defect, bone regeneration in the highest regenerative effects on orbital bone defects, compared to the other groups without BMP2. Biocoral scaffolds combined with BMSCs enhanced by BMP2 could improve the healing of critical-sized medial orbital wall defects in canines.

\section{Introduction}

The orbital bone plays an important role in the craniomaxillofacial region. The repair of orbital bone defects caused by trauma, cancer or infection, remains an immense challenge in reconstructive surgery. Large orbital bone defects usually do not heal spontaneously, and surgical intervention is often required. Although at present, autologous bone grafting is still considered the 'gold standard' approach for repairing orbital bone defects, this treatment has certain disadvantages, such as the significant morbidity at donor sites and the lack of sufficient material in certain patients (1-3). Therefore, alternatives approaches for the treatment of orbital bone defects are required.

Tissue engineering approaches have proven to be very effective in the repair of long bone defects in orthopaedics, with the use of large animals such as canines, goats and sheep as the model (4-10). An ideal tissue-engineered bone substitute should possess 3 elements: Osteoprogenitor cells, osteoinductive factors, and an osteoconductive scaffold, and is considered to be a potential substitute for autologous bone transplantation (11). Considering the irregular bone and the treatment of difficult defects, we focused our study on the orbital bone, as few studies have reported its regeneration using tissue engineering. Thus, we based our study on the repair of orbital bone defects using a modified tissue-engineered bone [a biocoral scaffold and bone marrow stromal cells (BMSCs)], as well as the human bone morphogenetic protein-2 (BMP2).

The aim of this study was to establish a canine orbital wall defect model simulating the large orbital bone defects in humans, and to determine whether tissue-engineered bone enhanced by BMP2 could be used to repair such a criticalsized orbital bone defect. A discovery such as this would be of great impact on the potential application of bone tissue engineering for the repair of large orbital bone defects. 


\section{Materials and methods}

Reagents. Adenoviruses containing human BMP2 cDNA (adv-hBMP2s) were kindly provided by Professor L. Jueren (University of Washington, St. Louis, MO). Adenoviruses containing LacZ cDNA (adv-LacZ) were obtained from the Department of Orthopedics, Ninth People's Hospital, Shanghai JiaoTong University School of Medicine, China. All animal experiments were conducted with the approval of the local Ethics Committee.

Preparation of coral scaffold. Biocoral scaffolds (from Beijing Invention Biology Engineering \& New Material Co., Ltd., Beijing, China) were molded into a 3-mm-high asymmetric cylinder with a $10-\mathrm{mm}$ diameter opening at 1 end and a 12-mm diameter opening at the other (Fig. 1), and were sterilized by ${ }^{60} \mathrm{Co}$ irradiation before use. The scaffolds had a volume porosity of $70 \%$ with a pore diameter of $300-350 \mu \mathrm{m}$. With a resistance to pressure of $300 \mathrm{MPa}$, they should be stable enough to fill bone gaps $(12,13)$.

Cell culture and gene transfection. A total of 16 adult Beagles (from the animal holding center at Shanghai JiaoTong University School of Medicine) in healthy condition, aged 18 months old and with an average weight of $20.9 \mathrm{~kg}$, were used in this study. The autologous bone marrow aspirates $(5 \mathrm{~mm})$ were harvested from the iliac crests of each canine, and the BMSCs were isolated and cultured as previously described (14). Briefly, the mononuclear cells were separated by Percoll (1.073 g/ml, Sigma, St Louis, MO) gradient centrifugation, and were plated in 100-mm dishes (Falcon, Franklin Lakes, $\mathrm{NJ})$ at a density of $1 \times 10^{5}$ cells $/ \mathrm{cm}^{2}$. The cells were cultured in basic culture medium containing $\alpha$ modification of Eagle's medium (Gibco, Grand Island, NY), 10\% fetal bovine serum (Gibco), $100 \mathrm{U} / \mathrm{ml}$ penicillin, $100 \mathrm{mg} / \mathrm{ml}$ streptomycin and $2.5 \mathrm{mg} / \mathrm{ml}$ fungizone (all from Sigma), at $37^{\circ} \mathrm{C}$ with $5 \% \mathrm{CO}_{2}$. The culture medium was changed after $48 \mathrm{~h}$ and then every 3 days. When the BMSCs reached $80-90 \%$ confluence, the passage 1 cells were detached with $0.25 \%$ trypsin/EDTA (Gibco). The passage 2BMSCs were transfected with a recombinant human BMP2 adenovirus overnight with a multiplicity of infection of $150 \mathrm{pfu} / \mathrm{cell}$, as previously reported $(9,15)$. The transduction efficiency of the cell population was evaluated by LacZ transduction and staining.

Real-time polymerase chain reaction. The expression of BMP2 incorporated into the transduced BMSCs was quantified by RT-PCR $24 \mathrm{~h}$ after gene transfection, as previously described $(16,17)$. Briefly, the cells were transferred into RNA lysis buffer solution and homogenized by gentle pipetting. The homogenized solution was purified using a Qiagen shredder column and total RNA was extracted with the RNeasy Mini Kit (Qiagen, Valencia, CA). The RNA samples were then reverse-transcribed to cDNA using Oligo dT primers and superscript III transcriptase. The final cDNA was then subjected to RT-PCR (7300 RT-PCR Real-Time PCR System; Applied Biosystems, Foster City, CA) to determine the expression of genes for BMP2, and the gene expression data were normalized to the expression of a housekeeping gene, glyceraldehyde-3-phosphatase dehydrogenase (GADPH). The primers used to amplify BMP2 and GAPDH were as follows: BMP2, (F) 5'-CTA GAC CTG TAT CGC AGG CAC T-3' and (R) 5'-CCC ACT CGT TTC TGG TAG TTC TT-3', 141 bp; GAPDH, (F) 5'-GAG CTG AAC GGG AAA CTC AC-3' and (R) 5'-CCC TGT TGC TGT AGC CAA AT-3', 304 bp.

Osteogenic differentiation of BMSCs after gene transduction. After gene transduction, the in vitro osteogenic differentiation of the cells was revealed by ALP staining on day 14 using the BM-purple method, and Alizarin Red staining on day 28 , as previously described (18).

Preparation of cell/coral composites. On day 5 after gene transduction, the BMSCs were detached from culture flasks (as described above), centrifuged to remove supernatant, and then resuspended in basic culture medium at a density of $2 \times 10^{7}$ cells $/ \mathrm{ml}$. The cells in suspension were slowly injected into the coral scaffolds using a syringe (500 $\mu 1 /$ scaffold $)$. After 4-h incubation to allow cell attachment, $20 \mathrm{ml}$ of the basic culture medium were then added to cover the composites. The BMSC/coral composites were subsequently cultured for 3 days in vitro before implantation.

Cell proliferation and BMP2 secretion of BMSCs on scaffolds. After 3-day incubation, a randomly chosen cell/scaffold composite was fixed in $2 \%$ formalin for $2 \mathrm{~h}$, cut into 2 halves, and then subjected for scanning electron microscopy (SEM) (Hitachi X-650, Tokyo, Japan) examination as previously described (19).

The cell proliferation of the BMSCs on the biocoral scaffolds was determined by DNA assay on days 3, 7, 14 and 21 after seeding, as described previously (19). Briefly, both the BMP2-transduced and non-transduced cells were collected from the scaffolds at different time-points and crushed for full lysis with proteinase $\mathrm{K}$ (Sigma) at $56^{\circ} \mathrm{C}$ overnight. The DNA content in the lysate was quantified spectrofluorometrically (523 nm) using Hoechst 33258 dye (Sigma), by correlating fluorescence with DNA content using standards containing known amounts of DNA. The scaffolds without seeding cells, soaked under the same conditions, were also measured to serve as the blank controls.

The level of BMP2 secreted from the BMP2-transduced BMSCs was also measured on days 3, 7, 14 and 21 after cell seeding using a custom ELISA kit (BMP2 ELISA kit, R\&D Systems Inc.) according to the manufacturer's instructions, and was divided by the total cell number of each sample, which was derived from the cell proliferation measurement. Again, BMSC/coral scaffolds and non-seeded scaffolds were used as the controls, respectively

Surgical procedure. The canines were anesthetized using a combination of ketamine $(35 \mathrm{mg} / \mathrm{kg}$ ) and xylazine $(5 \mathrm{mg} / \mathrm{kg})$, given subcutaneously. A $\mathrm{V}$-shaped incision provided access to the medial orbital wall. The orbital contents were moved to the opposite side, and a $10-\mathrm{mm}$ diameter trephine was used to remove the medial part of the orbital wall bone, leaving at least $5 \mathrm{~mm}$ to the orbital anterior rim. Then a dental bar cooled with sterile saline was used to create a $12-\mathrm{mm}$ diameter circular defect in the medial wall of both orbits (Fig. 1). The defects were treated with 1 of the following: adv-BMP2transfected BMSC/coral composite (group I, $\mathrm{n}=8$ ), BMSC/ 


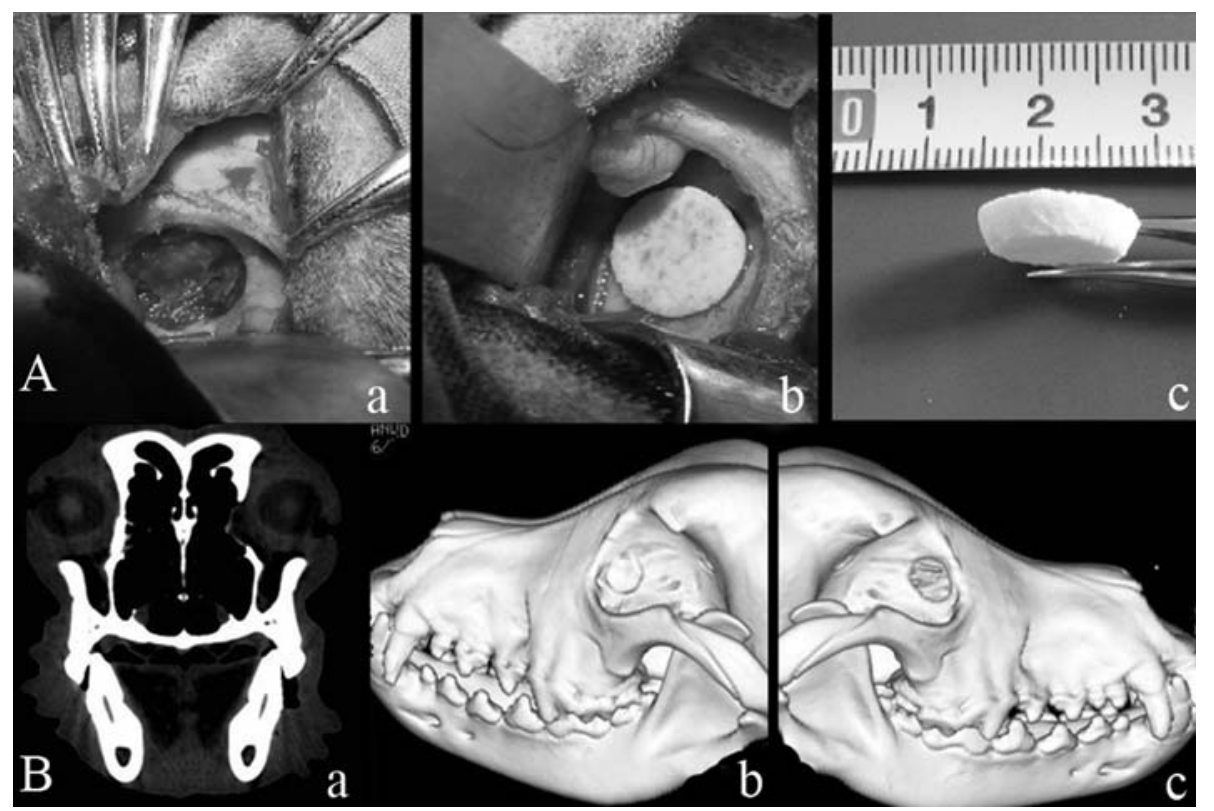

Figure 1. (A) (a) During the surgical procedure, an osteoperiosteal orbital wall defect of 12 mm in diameter, was made. Gross view of the removed orbital bone. (b) The defect was filled with a BMSC/coral construct. (c) Gross view of a coral scaffold. (B) CT images of the orbital defects post-implantation. (a) The coronal CT scans. (b) The left side defect was filled with coral. (c) The right side defect was not filled with anything.

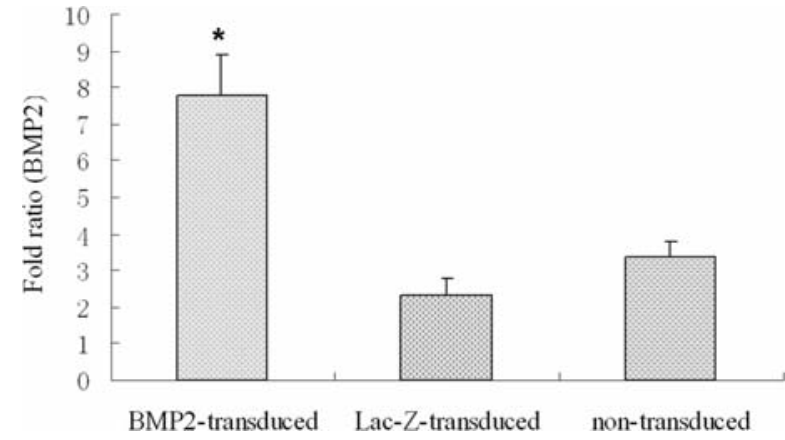

Figure 2. Quantitative gene expression of BMP2 for adv-BMP2-transduced, adv-LacZ-transduced and non-transduced BMSCs $24 \mathrm{~h}$ after gene transduction. Data were presented as a fold ratio after being normalized to GADPH expression. *Denotes a significantly higher gene expression $(\mathrm{p}<0.05)$ compared to the other groups. The error bars indicate $\mathrm{SD}, \mathrm{n}=4$.

coral composite (group II, $\mathrm{n}=8$ ), biocoral alone (group III, $\mathrm{n}=8$ ), or were left untreated (group IV, $\mathrm{n}=8$ ). The wound was then carefully closed in layers. Post-operatively, no subsequent dose of antibiotics was given. Each of the animals was housed in a pen and kept on a normal diet during the study. The unidirectional ocular mobility of the canines was evaluated by measuring passive eye movements in the superior, inferior, medial and lateral directions, using tweezers.

Gross observation and micro-computed tomography (micro$C T$ ) examination. Eight canines were sacrificed at 12 and 24 weeks, respectively, and 4 samples from each group were harvested at each time-point. The implants and surrounding tissues were dissected for gross observation and micro-CT examination ( $\mu$ CT80; Scanco Medical, Bassersdorf, Switzerland) as previously described (20). The CT settings used were as follows: Pixel matrix, 1024x1024; voxel size, $20 \mu \mathrm{m}$; slice thickness, $20 \mu \mathrm{m}$. From the reconstructed 3dimensional (3D) images, the percentage of the defect that was covered with new bone tissue (\% bone coverage), the total tissue volume $\left(\mathrm{mm}^{3}\right)$, bone volume $\left(\mathrm{mm}^{3}\right)$ and bone mineral density (BMD) $\left(\mathrm{mg} \mathrm{HA} / \mathrm{cm}^{3}\right)$, were calculated (21).

Deposition rate of new bone and histological analysis. In order to evaluate the new bone deposition rate, fluorescence bone labels were administered, as previously described $(22,23)$. Briefly, each canine was given an intramuscular injection of calcein $(20 \mathrm{mg} / \mathrm{kg})$ and tetracycline $(25 \mathrm{mg} / \mathrm{kg})$ (both from Sigma) on days 3 and 13, respectively, before sacrificing. After the canines were sacrificed, the specimens were fixed in $10 \%$ formalin solution and embedded in methylmethacrylate. All the tissues were cut lengthwise using a circular water-cooled diamond saw. The sections were visualized and photographed with an E-800 fluorescent microscope (Nikon) for the tetracycline labeling. The mean tetracycline (yellow) and calcein (green) double-labeling interval was determined by the Zeiss Axioplan Imaging System. The interval was divided by the administration time interval (10 days), and the mean value showed the rate of new bone deposition per day.

The sections were then surface-stained with Steven blue and van Gieson picro-fuchsin. The stained sections from each group were observed under a microscope and scanned using a 3-CCD video camera linked to an image processing system (Bioquant Osteo II V 8.10.20, Nashville, TN). Bone histomorphometric analysis of these stained sections was performed to quantitatively determine bone formation. The bone tissue area of each section was determined by dividing the total number of bone pixels by the total number of scaffold pixels based on color, using Image Pro Plus Software (Media Cybernetics, Silver Spring, MD) $(19,21)$. 

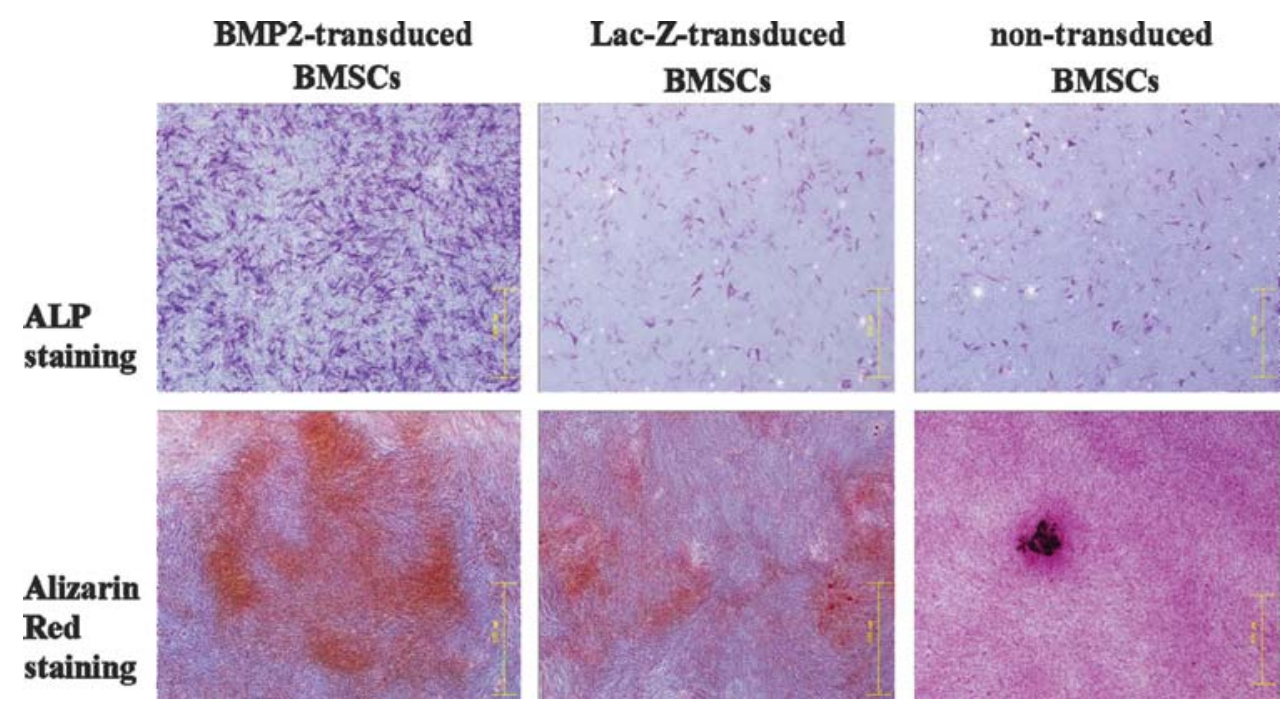

Figure 3. The osteogenic differentiation of BMP2-transduced BMSCs was evaluated by the staining of ALP on day 14, and Alizarin Red on day 28 after gene transduction (scale bars, $500 \mu \mathrm{m}$ ).
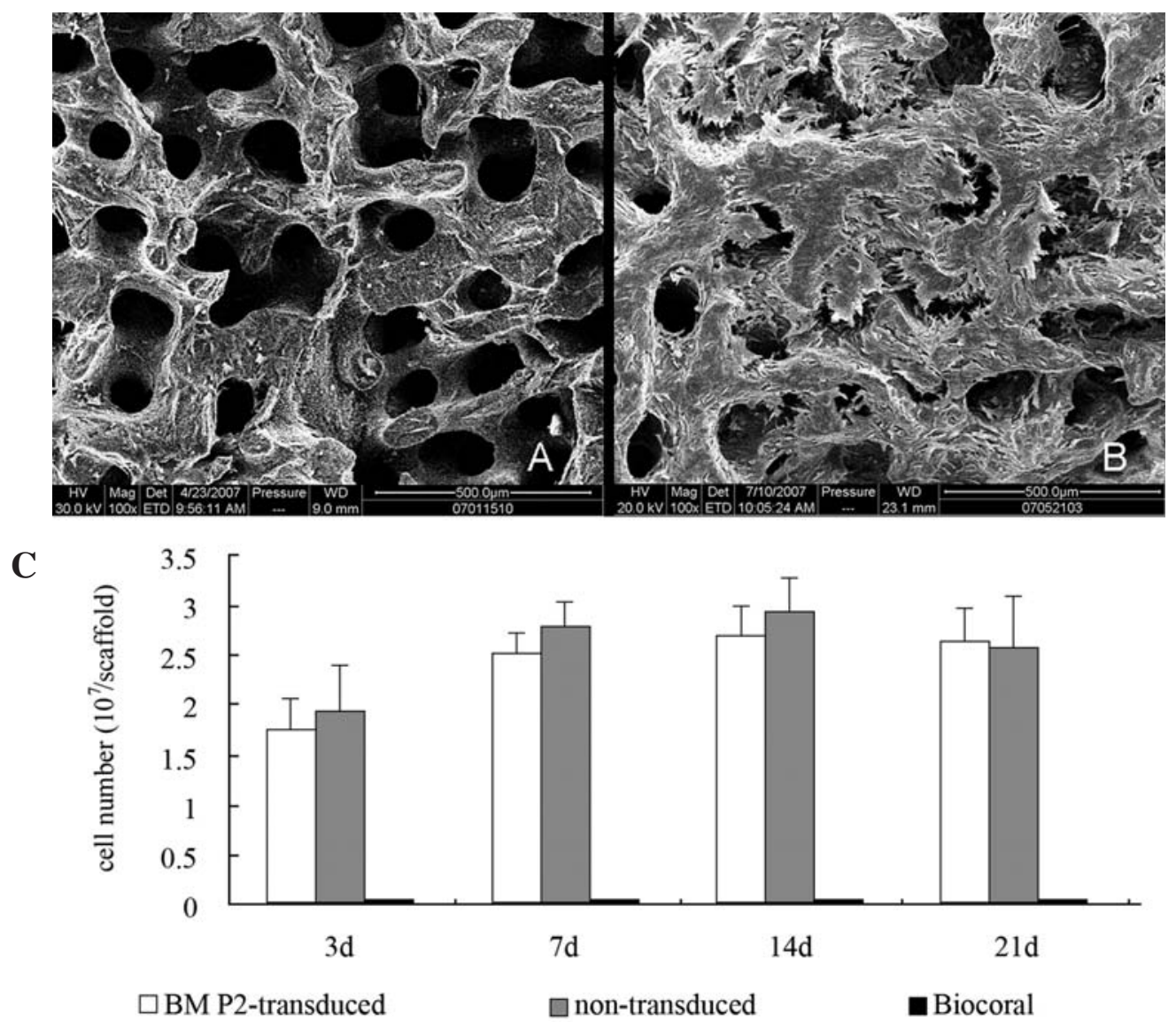

Figure 4. (A) SEM of a coral scaffold. (B) The BMSCs were seeded in the coral and they adhered and grew. In vitro growth and proliferation of BMP2transduced BMSCs on scaffolds. Three days after cell seeding, SEM observation revealed that dense ECMs associated with multilayer cells covered the pores of the coral scaffold (A), in contrast to the scaffold without any cells (B) (scale bars, $500 \mu \mathrm{m}$ ). (C) The cell numbers of the BMP2-transduced BMSCs and non-transduced BMSCs on the coral scaffolds were determined by DNA assay on days 3, 7,14 and 21. The cells of the 2 groups proliferated at a similar rate, and there was no significant difference in the cell number at each checked time-point ( $\mathrm{p}>0.05)$. The biocoral scaffolds alone cultured in the basic culture medium served as the blank control (the error bars indicate $\mathrm{SD}, \mathrm{n}=4$ ).

Statistical analysis. All data were expressed as the means \pm standard deviation (SD) with $\mathrm{n}=4$. Statistical analyses were performed with SPSS 16.0 (SPSS Inc., Chicago, IL). One- way ANOVA was used to analyze the statistical differences between 3 or 4 groups, and an independent Student's t-test was used to compare the parameters between 2 groups. For 

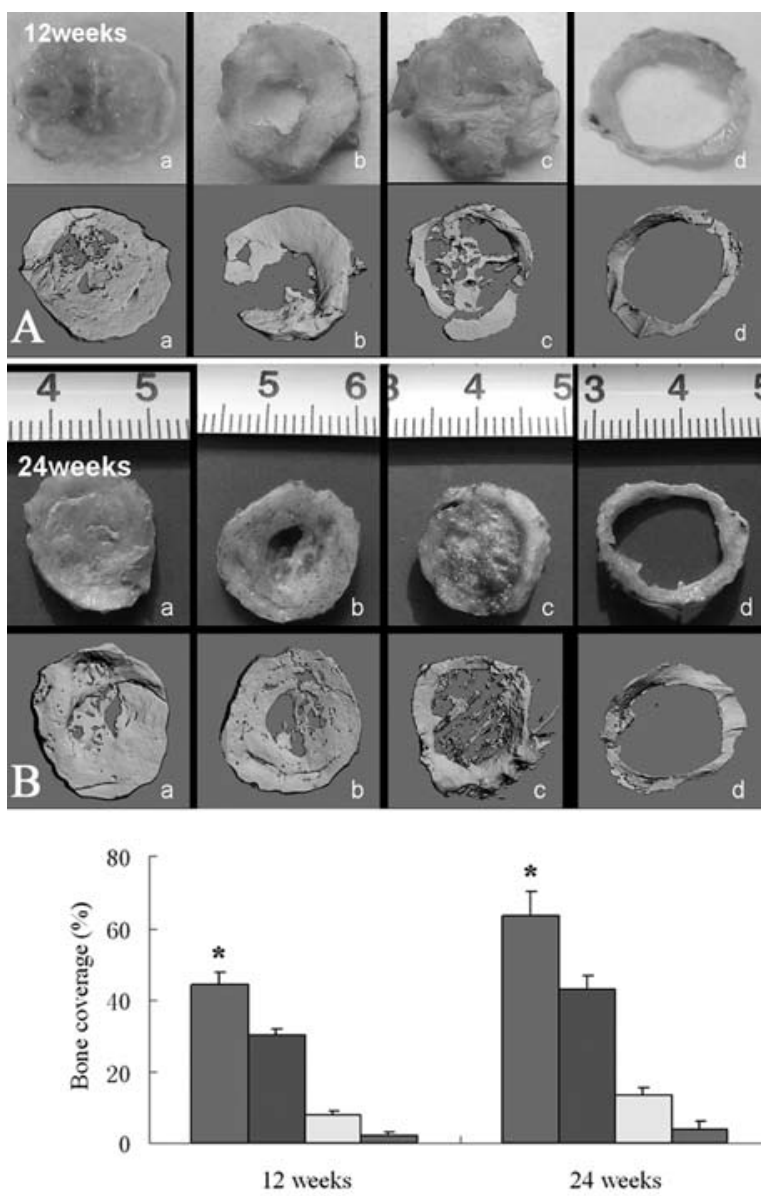

C
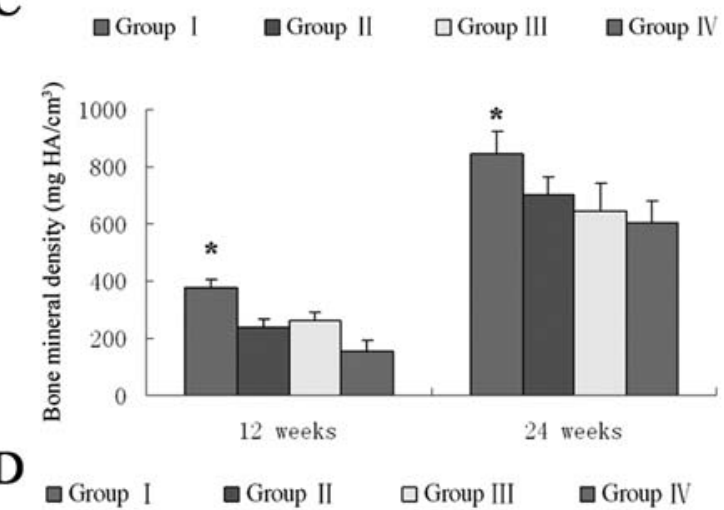

Figure 5. Gross view (top panel) and 3D micro-CT images (bottom panel) of the repaired orbital defects at 12 weeks (A) and 24 weeks (B) postimplantation (scale bars, $1 \mathrm{~mm}$ ). Quantitative micro-CT analysis revealed a statistically significant increase in bone coverage (C) and in the BMD of the newly regenerated bone (D) in group I $\left({ }^{*} \mathrm{p}<0.05\right)$, compared to the other groups at 12 and 24 weeks (the error bars indicate $\mathrm{SD}, \mathrm{n}=4$ ).

all the analyses, a p-value of $<0.05$ was considered statistically significant.

\section{Results}

Expression of BMP2 from gene-transfected BMSCs. The transduction efficiency of the BMSCs using ad-LacZ averaged at a value of $90 \%$, and the quantitative gene expression data for BMP2, are shown in Fig. 2. The results revealed a dramatic increase (2-3 fold) in the BMP2 gene expression for the
adv-BMP2-transduced BMSCs, which was significantly higher than that of the adv-LacZ-transduced and nontransduced BMSCs $(\mathrm{p}<0.05)$.

Osteogenic differentiation of BMSCs after gene transduction. The adv-BMP2-transduced BMSCs displayed a distinctly increased capability of osteogenic differentiation compared to the adv-LacZ-transduced and non-transduced cells over the observation period. As shown in Fig. 3, there was minimal blue discoloration of the cytoplasm of both the adv-LacZtransduced and non-transduced BMSCs. On the contrary, the adv-BMP2-transduced BMSCs were positively stained for ALP on day 14. A similar result was observed with the Alizarin Red staining on day 28 after gene transduction.

Cell proliferation and BMP2 secretion of BMSCs on scaffolds. After in vitro incubation for 3 days, the BMSC/coral composites were subjected to SEM examination just before implantation. As shown in Fig. 4A and B, compared to the biocoral scaffold alone, the pores were filled by cells including abundant extracellular matrices (ECMs) after 3 days of cell seeding, indicating that the scaffold had good compatibility.

By DNA assay, both cultures reached the stationary growth period between days 7 and 14. An increase in the cell number 2.7-fold was observed in the BMP2-expressing BMSC/ scaffold group after 14 days of culture, and 2.9-fold in the non-transduced BMSC/coral group (Fig. 4C). There was no significant difference in the cell number between these 2 groups at each checked time-point ( $\mathrm{p}>0.05)$.

In order to further address whether the BMP2-expressing potential of the BMSCs grown in the scaffolds could be maintained, the release of BMP2 was measured on days 3, 7, 14 and 21 , post-seeding. The ELISA measurement showed that the BMSCs were able to synthesize, process, and secrete human BMP2 following transduction with adv-BMP2, with a consistently high production of the BMP2 protein $\left(27.6 \pm 3.5 \mathrm{ng} / 10^{6}\right.$ cells $/ 24 \mathrm{~h}$ ) being achieved over 3 weeks. In contrast, the nontransduced BMSCs secreted BMP2 at a level of $3.13 \pm 0.32 \mathrm{ng} /$ $10^{6}$ cells $/ 24 \mathrm{~h}$, which was significantly lower than that of the BMP2-transduced cells over the observation period $(\mathrm{p}<0.001)$.

Gross observation and micro-CT examination. All of the wounds healed uneventfully without infection, and all the animals survived until the day of sacrifice. Eye-globe mobility was not influenced after surgery. In order to follow-up the new bone regeneration and the development of bone union within the orbital defects, gross observation and micro-CT measurements were taken at 12 and 24 weeks post-operation. Representative images from each group are shown in Fig. 5. In group I, almost $50 \%$ of the defect was filled with newly formed bone tissue at 12 weeks, and almost complete bone union was achieved at 24 weeks. The interface between native bone and regenerated bone became indistinct, and a continuous layer of mucous membrane on the side of the ethmoid sinus could be observed. Incomplete bone bridging of the defect was also found in group II, but the bone coverage was significantly lower than that of group I at these 2 time-points. On the contrary, non-union was observed in group III (the biocoral only group). The scaffold was degraded almost completely at 12 weeks, and most of the original defect was 


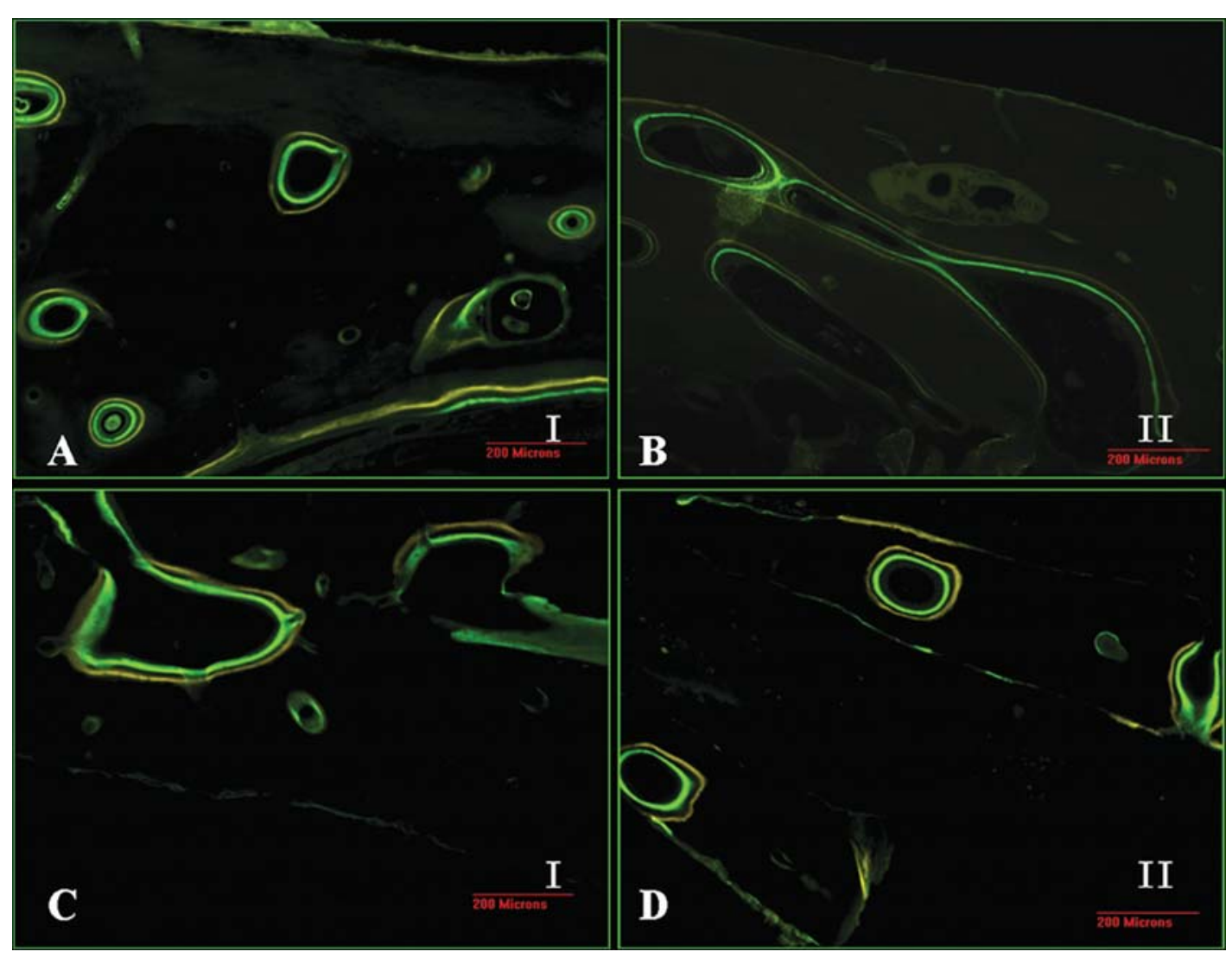

Figure 6. Observation of the new bone deposition by tetracycline/calcein fluorescent labeling at 12 (A and B), and 24 weeks post-implantation (C and D) of groups I and II (scale bars, $200 \mu \mathrm{m}$ ).

covered with soft tissues at 24 weeks. In group IV, non-union also occurred. The fibrous tissue occupied the defect area, and only a trivial amount of new bone formed at the edge of the defects.

Deposition rate of new bone and histological analysis. As no obvious bone formation occurred in groups III and IV, tetracycline/calcein fluorescent labeling was only observed in groups I and II. Representative fluorescence images of the defect sites are shown in Fig. 6. At 12 weeks, group I attained

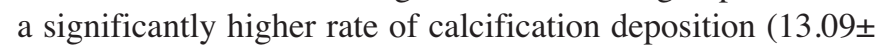
$2.38 \mu \mathrm{m} /$ day $)$ than that of Group II $(9.07 \pm 1.23 \mu \mathrm{m} /$ day $)$ $(\mathrm{p}=0.001)$. At 24 weeks, the new bone deposition rate of group I $(9.54 \pm 1.83 \mu \mathrm{m} /$ day $)$ was still higher than that of group II $(8.82 \pm 1.60 \mu \mathrm{m} /$ day $)$. However, the difference was not significant $(\mathrm{p}>0.05)$.

The presence of bone regeneration within the orbital defects was performed through histological analysis at 12 and 24 weeks after implantation, respectively. At 12 weeks, gross qualitative histological analysis of whole sections suggested that the implantation of the BMP2-modified BMSC/coral composites (group I ) resulted in the formation of more bone tissue compared to group II (with no BMP2) (Fig. 7A and B). High magnification images showed that the newly formed bone tissue had a woven, trabecular appearance in both groups, with the scaffolds being almost completely absorbed. The quantitative histomorphometric analysis of bone formation revealed a significant increase in group I $(62.03 \pm 3.32 \%)$ relative to bone formation in group II
$(34.68 \pm 2.75 \%)(\mathrm{p}<0.01)$. At 24 weeks post-implantation, greater osseous bridging of the circular orbital defect was found in group I $(74.63 \pm 7.94 \%)$, whereas group II only exhibited partial bone bridging $(50.21 \pm 3.92 \%)$, which was still much less compared to that in group I $(\mathrm{p}<0.05)$. Group II also revealed remodeled, mature areas of bone regions, similar to group I. However, a clear boundary between newly formed tissue-engineered and native bone could still be found in group II. As there was no bone union in groups III and IV, histological examination was not applied in these groups.

\section{Discussion}

In our study, we examined the bone regeneration ability of orbital defect under the following 3 conditions: BMP2 transduced BMSC/coral composites, non-transduced BMSC/coral composites and coral scaffolds alone. These were compared in a Beagle model. Our results showed that the critical-sized bone defect of the canine orbital wall could be repaired by biodegradable coral scaffolds combined with BMP2-transduced autologous BMSCs, as opposed to the non-transduced $\mathrm{BMSC} /$ coral composites and the scaffold group.

The human orbital wall exhibits unique anatomical properties, as its inner side makes up the ethmoid sinus, and its outer side contains the soft tissue. It has been reported that orbital bone defects larger than $2 \mathrm{~cm}^{2}$ cannot heal spontaneously, and this is why surgical therapies are required (24). We previously investigated the skull anatomical characteristics of canines, goats, and rabbits and found that the 

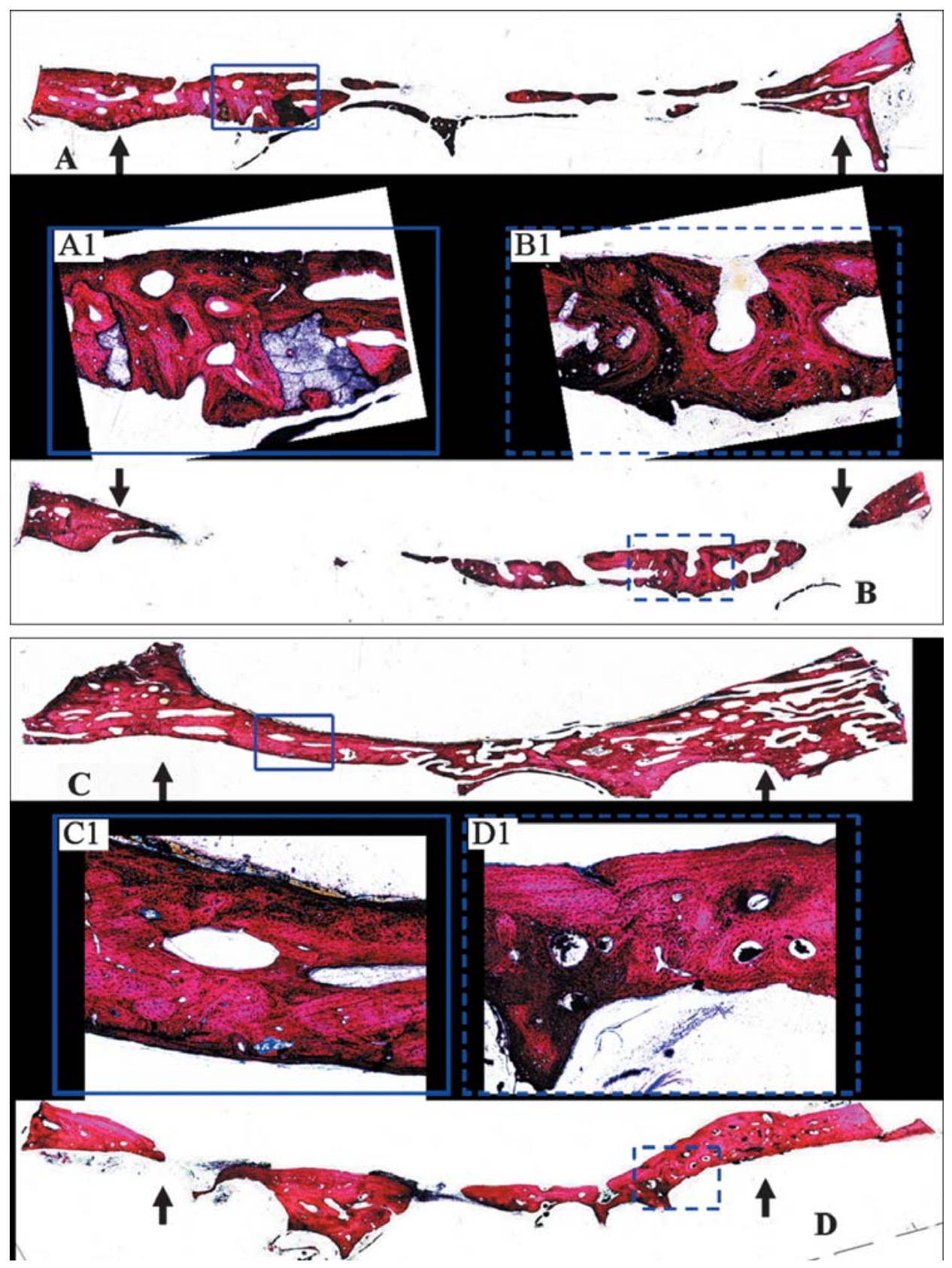

Figure 7. Histological observation of the repaired orbital defects in groups I and II at 12 and 24 weeks post-implantation. More new bone formation was observed in group I (A) than in group II (B) at 12 weeks, and almost complete bone union was achieved between the edges of the defect in group I at 24 weeks (C). The continuity across the defect was not restored totally in group II at 24 weeks (D). Detailed views of the regenerated bone in group I (solid frame, A1 and C1) and Group II (dotted frame, B1 and D1) were taken at higher magnification (x100). Compared to 12 weeks, more mature and organized bone tissue formed in both groups at 24 weeks. (The arrows indicated the interfaces between the implant and host bone). Histological observation of bone regeneration at 3 and 6 months post-implantation. In group A, more new bone was observed compared to group B. Almost complete bone union was achieved between the edges of the defects in group A. The continuity across the defects was not restored totally in group B.

canine medial orbital wall is more structurally similar to that of humans. Furthermore, we set up a $10-\mathrm{mm}$ circular defect model in the canine medial orbital wall, and found that it cannot heal without any treatment even after 6 months.

Consequently, in this study, the canine model was chosen and a 12-mm circular defect of the medial orbital wall was made. The results from our current study, using the canine as opposed to other animal modes, could provide stronger evidence supporting the clinical application of tissue engineering for the treatment of critical sized orbital defects.
Indeed, there have been many studies that use materials to repair orbital defects. Due to the limitations of autografts and allografts, orbital bone defects are treated by implanting suitable biomaterials to bridge the defect area and to limit the herniation of the orbital contents (25-29). The materials frequently used for reconstruction of the orbit include metals, ceramics, and synthetic polymers. However, questions still exist. Non-resorbable materials, such as titanium and silicone, however, do not allow for the in-growth and perfusion of fibrous tissue and bone, and remain as foreign 
bodies in the implant area, which makes them more susceptible to infection, extrusion, migration, without the potential for replacement by host tissue (30-32). Also the application of resorbable materials is limited by their significant induction of foreign-body reactions and the lack of osteoconductive characteristics, and thus they also have minimal potential for new bone growth (33-35). Therefore, the selection of a scaffold is an important point in the process of orbital defect repair using tissue engineering.

The premature degradation of a scaffold can destroy the tissue structure if new bone has not been generated yet, while the lingering degradation of scaffold can also affect the repair process, such as tissue structure remodelling $(14,20)$. Therefore, an optimal scaffold for bone engineering should have a suitable degradation rate that can well match the speed of new bone formation. In our study, a biocoral scaffold was used which showed a good degradation rate that could well match the new bone formation rate in the application of bone tissue engineering $(7,8,12,13)$. In this study, when combined with BMSCs, the new bone formation became obvious at 12 weeks post-operation and the coral had been almost completely degraded at the same time. However, in group III (the biocoral only group), the migration rate of native osteoprogenitor cells was not fast enough to match the degradation of the scaffold. Thus, only fibrous union was achieved. Hence, our results also suggest that native coral alone as a bone graft substitute was ineffective for healing a critical-sized bone defect in the medial orbital wall.

In addition, in our study, BMP2 was used to enhance osteogenesis. As one of the BMP family members, BMP2 has been used to induce bone formation and to repair bone defects in various animal models (9,36-41). The results of this study even ascertain the importance of using BMSCs transfected by BMP2 for the repair of critical-sized defects of the canine orbital wall. However, our study also showed the application of tissue engineering for the treatment of orbital bone defects. Thus, compared to the BMSCs alone, we showed that bone healing could be accelerated by BMP2-expressing BMSCs. Bostrom, et al previously found that, after treatment with BMP2, $25 \%$ of the non-unions required a secondary bone graft procedure (42). Kujala, et al contended that BMP was incapable of reaching target cells secondary to inadequately vascularized tissue (43). Moreover, according to our previous study, a $10-\mathrm{mm}$ circular bone defect of the canine orbital wall did not heal spontaneously (a critical-sized defect) due to the disruption of the adjacent bone vascularity. As angiogenesis precedes osteogenesis in skeletogenesis as well as in fracture healing (44), further studies of constructing a pre-vascularized tissue-engineered bone graft for improving the repair of orbital defects, are required.

In summary, the results of the present study indicate that a biocoral scaffold combined with BMSCs enhanced by BMP2 can repair the critical-sized orbital defects in a canine model, suggesting that this method could be a feasible approach for the clinical reconstruction of orbital bone defects.

\section{Acknowledgements}

This study was supported by the Shanghai Leading Academic Discipline Project (no. S30205) the National Natural Science
Foundation of China (nos. 30571879 and 30973279), the National Key Program for Basic Research of China (no. 2010CB529902), the Ph.D. Programs Foundation of the Ministry of Education of China (no. BXJ0826), and the Science and Technology Commission of Shanghai (nos. 08ZR1412900 and 10411964000).

\section{References}

1. Younger EM and Chapman MW: Morbidity at bone graft donor sites. J Orthop Trauma 3: 192-195, 1989.

2. Sasso RC, Williams JI, Dimasi N, et al: Postoperative drains at the donor sites of iliac-crest bone grafts. A prospective, randomized study of morbidity at the donor site in patients who had a traumatic injury of the spine. J Bone Joint Surg Am 80: 631-635, 1998.

3. Grob D: [Problems at the donor site in autologous bone transplantation]. Unfallchirurg 89: 339-345, 1986.

4. Bruder SP, Kraus KH, Goldberg VM, et al: The effect of implants loaded with autologous mesenchymal stem cells on the healing of canine segmental bone defects. J Bone Joint Surg Am 80: 985-996, 1998.

5. Kon E, Muraglia A, Corsi A, et al: Autologous bone marrow stromal cells loaded onto porous hydroxyapatite ceramic accelerate bone repair in critical-size defects of sheep long bones. $J$ Biomed Mater Res 49: 328-337, 2000.

6. Arinzeh TL, Peter SJ, Archambault MP, et al: Allogeneic mesenchymal stem cells regenerate bone in a critical-sized canine segmental defect. J Bone Joint Surg Am 85-A: 1927-1935, 2003.

7. Zhu L, Liu W, Cui L, et al: Tissue-engineered bone repair of goat-femur defects with osteogenically induced bone marrow stromal cells. Tissue Eng 12: 423-433, 2006.

8. Petite H, Viateau V, Bensaid W, et al: Tissue-engineered bone regeneration. Nat Biotechnol 18: 959-963, 2000.

9. Dai KR, Xu XL, Tang TT, et al: Repairing of goat tibial bone defects with BMP2 gene-modified tissue-engineered bone. Calcif Tissue Int 77: 55-61, 2005.

10. Kuhne M, John T, El-Sayed K, et al: Characterization of auricular chondrocytes and auricular/articular chondrocyte cocultures in terms of an application in articular cartilage repair. Int J Mol Med 25: 701-708, 2010

11. Hou R, Chen F, Yang Y, et al: Comparative study between coral-mesenchymal stem cells-rhBMP2 composite and autobone-graft in rabbit critical-sized cranial defect model. J Biomed Mater Res A 80: 85-93, 2007.

12. Louisia S, Stromboni M, Meunier A, et al: Coral grafting supplemented with bone marrow. J Bone Joint Surg Br 81: 719-724, 1999.

13. Geiger F, Lorenz $\mathrm{H}, \mathrm{Xu} \mathrm{W}$, et al: VEGF producing bone marrow stromal cells (BMSC) enhance vascularization and resorption of a natural coral bone substitute. Bone 41: 516-522, 2007.

14. Yuan J, Cui L, Zhang WJ, et al: Repair of canine mandibular bone defects with bone marrow stromal cells and porous betatricalcium phosphate. Biomaterials 28: 1005-1013, 2007.

15. Li H, Dai K, Tang $\mathrm{T}$, et al: Bone regeneration by implantation of adipose-derived stromal cells expressing BMP2. Biochem Biophys Res Commun 356: 836-842, 2007.

16. Sobajima S, Shimer AL, Chadderdon RC, et al: Quantitative analysis of gene expression in a rabbit model of intervertebral disc degeneration by real-time polymerase chain reaction. Spine J 5: 14-23, 2005.

17. Livak KJ and Schmittgen TD: Analysis of relative gene expression data using real-time quantitative PCR and the 2(-Delta Delta C(T)) method. Methods 25: 402-408, 2001.

18. Liu G, Zhou H, Li Y, et al: Evaluation of the viability and osteogenic differentiation of cryopreserved human adiposederived stem cells. Cryobiology 57: 18-24, 2008.

19. Liu G, Shu C, Cui L, et al: Tissue-engineered bone formation with cryopreserved human bone marrow mesenchymal stem cells. Cryobiology 56: 209-215, 2008.

20. Liu G, Zhao L, Zhang W, et al: Repair of goat tibial defects with bone marrow stromal cells and beta-tricalcium phosphate. J Mater Sci Mater Med 19: 2367-2376, 2008.

21. Kaigler D, Wang Z, Horger K, et al: VEGF scaffolds enhance angiogenesis and bone regeneration in irradiated osseous defects. J Bone Miner Res 21: 735-744, 2006.

22. Ejersted C, Andreassen TT, Hauge EM, et al: Parathyroid hormone (1-34) increases vertebral bone mass, compressive strength, and quality in old rats. Bone 17: 507-511, 1995. 
23. Ma B, Sampson W, Wilson D, et al: A histomorphometric study of adaptive responses of cancellous bone in different regions in the sheep mandibular condyle following experimental forward mandibular displacement. Arch Oral Biol 47: 519-527, 2002.

24. Parsons GS and Mathog RH: Orbital wall and volume relationships. Arch Otolaryngol Head Neck Surg 114: 743-747, 1988.

25. Ilankovan V and Jackson IT: Experience in the use of calvarial bone grafts in orbital reconstruction. Br J Oral Maxillofac Surg 30: 92-96, 1992.

26. Kontio RK, Laine P, Salo A, et al: Reconstruction of internal orbital wall fracture with iliac crest free bone graft: clinical, computed tomography, and magnetic resonance imaging followup study. Plast Reconstr Surg 118: 1365-1374, 2006.

27. Gazdag AR, Lane JM, Glaser D, et al: Alternatives to Autogenous Bone Graft: Efficacy and Indications. J Am Acad Orthop Surg 3: 1-8, 1995.

28. Ozaki W and Buchman SR: Volume maintenance of onlay bone grafts in the craniofacial skeleton: micro-architecture versus embryologic origin. Plast Reconstr Surg 102: 291-299, 1998

29. Magdolen U, Auernheimer J, Dahmen C, et al: Growth promoting in vitro effect of synthetic cyclic RGD-peptides on human osteoblast-like cells attached to cancellous bone. Int J Mol Med 17: 1017-1021, 2006

30. Gear AJ, Lokeh A, Aldridge JH, et al: Safety of titanium mesh for orbital reconstruction. Ann Plast Surg 48: 1-9, 2002.

31. Jacono AA and Moskowitz B: Alloplastic implants for orbital wall reconstruction. Facial Plast Surg 16: 63-68, 2000.

32. Ellis E III and Messo E: Use of nonresorbable alloplastic implants for internal orbital reconstruction. J Oral Maxillofac Surg 62: 873-881, 2004.

33. Rohner D, Hutmacher DW, Cheng TK, et al: In vivo efficacy of bone-marrow-coated polycaprolactone scaffolds for the reconstruction of orbital defects in the pig. J Biomed Mater Res B Appl Biomater 66: 574-580, 2003.

34. Potter JK and Ellis E: Biomaterials for reconstruction of the internal orbit. J Oral Maxillofac Surg 62: 1280-1297, 2004.
35. Kontio R, Suuronen R, Salonen O, et al: Effectiveness of operative treatment of internal orbital wall fracture with polydioxanone implant. Int J Oral Maxillofac Surg 30: 278-285, 2001.

36. Asamura S, Mochizuki Y, Yamamoto M, et al: Bone regeneration using a bone morphogenetic protein-2 saturated slow-release gelatin hydrogel sheet: evaluation in a canine orbital floor fracture model. Ann Plast Surg. 64: 496-502, 2010.

37. Lieberman JR, Daluiski A and Einhorn TA: The role of growth factors in the repair of bone. Biology and clinical applications. J Bone Joint Surg Am 84-A: 1032-1044, 2002.

38. Tsuchida H, Hashimoto J, Crawford E, et al: Engineered allogeneic mesenchymal stem cells repair femoral segmental defect in rats. J Orthop Res 21: 44-53, 2003.

39. Baltzer AW, Lattermann C, Whalen JD, et al: Potential role of direct adenoviral gene transfer in enhancing fracture repair. Clin Orthop Relat Res: S120-S125, 2000.

40. Xu M, Dai W and Deng X: Effects of magnesium sulfate on brain mitochondrial respiratory function in rats after experimental traumatic brain injury. Chin J Traumatol 5: 361-364, 2002.

41. Clement JH, Raida M, Sanger J, et al: Bone morphogenetic protein 2 (BMP2) induces in vitro invasion and in vivo hormone independent growth of breast carcinoma cells. Int J Oncol 27: 401-407, 2005.

42. Bostrom MP, Saleh KJ and Einhorn TA: Osteoinductive growth factors in preclinical fracture and long bone defects models. Orthop Clin North Am 30: 647-658, 1999.

43. Kujala S, Raatikainen T, Ryhanen J, et al: Composite implant of native bovine bone morphogenetic protein (BMP) and biocoral in the treatment of scaphoid nonunions-a preliminary study. Scand J Surg 91: 186-190, 2002.

44. Glowacki J: Angiogenesis in fracture repair. Clin Orthop Relat Res: S82-S89, 1998. 